\title{
Effect of different salivary exposure times on the rehardening of acid-softened enamel
}

\section{Catarina Ribeiro Barros de ALENCAR ${ }^{(a)}$ \\ Fernanda Lyrio MENDONÇA(a) \\ Luísa Belluco GUERRINI(a) \\ Maisa Camillo JORDÃO(a) \\ Gabriela Cristina de OLIVEIRA(a) \\ Heitor Marques HONÓRIO(a) \\ Ana Carolina MAGALHÃES(b) \\ Daniela RIOS(a)}

(a) Universidade de São Paulo - USP, Bauru School of Dentistry, Department of Pediatric Dentistry, Orthodontics and Public Health, Bauru, SP, Brazil;

(b) Universidade de São Paulo - USP, Bauru School of Dentistry, Department of Biological Sciences, Bauru, SP, Brazil.
Declaration of Interests: The authors certify that they have no commercial or associative interest that represents a conflict of interest in connection with the manuscript.

Corresponding Author:

Daniela Rios

E-mail: daniriosop@yahoo.com.br

DOI: 10.1590/1807-3107BOR-2016.vol30.0104

Submitted: Nov 15, 2015

Accepted for publication: May 11, 2016

Last revision: July 8, 2016

\begin{abstract}
This in situ study assessed the effect of different times of salivary exposure on the rehardening of acid-softened enamel. Bovine enamel blocks were subjected in vitro to a short-term acidic exposure by immersion in $0.05 \mathrm{M}$ ( $\mathrm{pH}$ 2.5) citric acid for $30 \mathrm{~s}$, resulting in surface softening. Then, 40 selected eroded enamel blocks were randomly assigned to 10 volunteers. Intraoral palatal appliances containing 4 enamel blocks were constructed for each volunteer, who wore the appliance for 12 nonconsecutive hours: initial $30 \mathrm{~min}$, followed by an additional 30, and then by an additional 1 hour. For the last additional 10 hours the appliances were used at night, during the volunteers' sleep. Surface hardness was analyzed in the same blocks at baseline, after erosion and after each period of salivary exposure, enabling percentage of surface hardness recovery calculation (\%SHR). The data were tested using repeated measures ANOVA and Tukey's test $(\alpha=0.05)$. Increasing periods of salivary action promoted a progressive increase in the surface hardness $(p<0.001)$. However a similar degree of enamel rehardening $(p=0.641)$ was observed between 2 hours $(49.9 \%)$ and 12 hours (53.3\%) of salivary exposure. Two hours of salivary exposure seems to be appropriate for partial rehardening of the softened enamel surface. The use of the intraoral appliance during sleep did not improve the enamel rehardening after erosion.
\end{abstract}

Keywords: Tooth Erosion; Dental Enamel; Saliva; Tooth Remineralization.

\section{Introduction}

Dental erosion is the softening effect of an acid on the tooth surface, while the subsequent tissue loss induced by mechanical forces is called erosive tooth wear. ${ }^{1,2}$ The occurrence and progression of dental erosion depend on the interaction between behavioral, chemical and biological factors. ${ }^{1}$ Among the factors that influence the pathogenesis of dental erosion, the protective potential of saliva has been described in several studies. ${ }^{3,4}$

After the intake of an acidic food or drink, saliva acts as a diluting agent, clearing the remnants of acids from the mouth. Salivary flow, pellicle and buffer capacity contribute to the protective effect of saliva. ${ }^{5}$ Furthermore, saliva has the ability to reduce demineralization rate and enhance remineralization by providing calcium, phosphate and fluoride to eroded enamel and dentine. ${ }^{4}$ Thus, saliva can theoretically protect enamel against dental erosion in several ways, but the effectiveness of this protective capacity is unclear. ${ }^{6}$ 
Studies designed to identify the individual erosive potential of products or to assess the effectiveness of preventive methods should ideally be conducted in $v i v o^{7}$, under the natural effect of saliva. However, in vivo studies do not allow an accurate erosive tooth wear measurement and evaluation of lesion progression pattern, and require long interventions. ${ }^{7}$ To overcome these challenges, in vitro and in situ models are used. In situ models offer the advantage of allowing a controlled erosive challenge and expose specimens to the oral environment. ${ }^{7,8}$ Nevertheless, in situ studies that consider saliva a remineralizing agent do not specify the time required for remineralization to occur in order to simulate clinical conditions. While some studies recommend the continuous use of intraoral appliances, which are removed from the oral cavity only for eating and for oral hygiene, ${ }^{9}$ other studies establish the use of intraoral devices only during the day. ${ }^{10}$ There are no reports regarding the standardization of the time needed to simulate the remineralization of the eroded surface between the erosive challenges. ${ }^{11}$ Because the in situ time required for saliva to achieve its maximum repair potential after an erosive challenge is not well defined, every research group adopts its own specific protocol. In addition, knowledge about the effect of overnight use of intraoral devices on enamel rehardening is incomplete. The absence of standardization hinders meaningful comparisons among different researches and extrapolation of in situ findings into clinical situations.

Taking these aspects into consideration, the aim of this study was to evaluate the effect of different salivary exposure times on the rehardening of initially eroded enamel. The null hypothesis was that different times of human saliva exposure has no effect on the enamel rehardening.

\section{Methodology}

\section{Experimental design}

Enamel was evaluated in situ after 30 minutes, 1, 2 and 12 hours of salivary exposure. Enamel blocks with artificial initial erosion lesion $(n=40)$ were randomly divided among volunteers $(n=10)$, who wore intraoral palatal appliances during 12 nonconsecutive hours. The response variable was superficial hardness recovery (SHR), which was measured in the same blocks at baseline, after erosion and after each period of salivary exposure.

\section{Enamel samples preparation}

One hundred enamel blocks $(4 \times 4 \times 3 \mathrm{~mm})$ were prepared from the labial surfaces of bovine incisor crowns. The blocks were cut using an ISOMET low speed saw (Buehler Ltd., Lake Bluff, IL, USA) with two diamond disks (Extec Corp., Enfield, CT, USA), which were separated by a $4-\mathrm{mm}$ thick spacer. The blocks' surfaces were ground flat with water-cooled silicon carbide discs $(320,600$, and 1200 grade papers; Buehler, Ltd., Lake Bluff, IL, USA), and polished with felt paper sprayed with $1 \mu \mathrm{m}$ diamond spray (Buehler, Ltd., Lake Bluff, IL, USA). The blocks were cleaned using an ultrasonic device for 2 minutes and inspected for presence of white spots and cracks using a microscope $(\times 40)$. The samples were sterilized by ethylene oxide.

The baseline surface hardness (SHi) was determined using the average values of five indentations performed at $100 \mu \mathrm{m}$ distances from each other (Knoop diamond, $25 \mathrm{~g}, 10 \mathrm{~s}$, Hardness tester from Buehler). Eighty blocks were selected by the initial surface hardness (mean SHi value $=343.9 \pm 21.4 \mathrm{KHN}$ ) to be demineralized in vitro (initial erosion lesion). The number of blocks was twice the required amount, to allow discard of non-standard demineralized blocks.

\section{Initial erosion lesion}

The erosion lesion was established following the definition by Young and Tenuta ${ }^{11}$, in which the initial erosion corresponds to the very first tooth surface changes caused by short-term acidic exposures before any tissue loss is observed. A pilot in vitro study showed that the enamel immersion in $0.05 \mathrm{M}(\mathrm{pH} 2.5)$ citric acid solution for 30 seconds was the optimum time for the development of initial erosion lesions, as longer periods caused enamel loss and difficulty in viewing baseline indentations. Blocks were, therefore, demineralized under those conditions, with agitation (Flatbed oscillator, $60 \mathrm{rpm}$ ). The surface hardness after demineralization (SHd) was measured at $100 \mu \mathrm{m}$ distance from the SHi, to obtain the degree of softening. Enamel samples presenting the percentage of surface hardness change $(\% \mathrm{SHC}=[(\mathrm{SHi}-\mathrm{SHd}) /(\mathrm{SHi})] \times 100)$ between 30 and $40 \%$ were selected $(n=40)$ and randomly allocated 
to the volunteers by a second researcher using Excel software (Microsoft Office 2013, USA).

\section{Volunteers and in situ phase}

This study received ethical approval from the local Institutional Ethics Committee (protocol $\left.n^{\circ} 141.316 / 2012\right)$, and was conducted in full accordance with the Declaration of Helsinki. Ten healthy adult subjects (eight females and two males, aged 19-30 years) who fulfilled the inclusion criteria (residing in the same fluoridated area $-0.70 \mathrm{mg} F / 1$ ), physiological stimulated salivary flow rate $>1 \mathrm{~mL} / \mathrm{min}$, adequate oral health - no caries or erosion lesions), without violating the exclusion criteria (systemic illness, pregnancy or breastfeeding, under orthodontic intervention, use of supplementary fluoride compounds in the last two months) took part in this study, after given an informed written consent.

Sample size calculation was based on a pilot study with 4 volunteers. A sample size of 10 volunteers was estimated based on an a-error of $5 \%, \beta$-error of $20 \%, 14.8$ estimated standard deviation and $25.0 \%$ minimum detectable difference in means.

The intraoral upper arch palatal appliances were made with acrylic resin on plaster models of each volunteer. The appliance had two vertical rows, on the right and left sides, containing two cavities $(6 \times 6 \times 3 \mathrm{~mm})$ in each side, for the enamel blocks fixation. The enamel blocks were fixed with wax and were carefully levelled with the appliance surface in order to avoid dental plaque accumulation.

Seven days prior to the experiment, volunteers brushed their teeth with standard fluoride toothpaste (Total 12, 1.100 ppm F, Colgate, Brazil) 3 times a day, and they were also instructed to avoid use of any other fluoride-containing product. During the in situ phase, tooth brushing was performed 1 hour before each period of salivary exposure.

Volunteers were asked to avoid touching the enamel blocks with the tongue during appliance use. After salivary exposure (30 minutes, 1 hour, 2 hours and 12 hours), the volunteers removed the intraoral appliance (without washing) and the enamel blocks were subjected to surface hardness evaluation. Between the surface hardness measurements, special care was taken to avoid cross-contamination, including individualized use of gloves for handling each intraoral appliance, and decontamination of the Knoop diamond of the Hardness tester after each measurement. After each measurement, blocks were repositioned in their original cavities, and the appliance was worn until the next measurement. During the 3 hours required for surface hardness evaluation and fixation of the blocks in the intraoral appliances, volunteers had meals or snacks and brushed their teeth, and after one hour, the appliances were reinstalled. When not in use, the appliances were kept in a plastic box, wrapped in gauze moistened in water (Bauru, São Paulo, Brazil; 0.7 ppm F). For the last evaluated period, the appliances were used for an additional 10 hours at night, during sleep. Volunteers and researchers were not blind to the study protocol. Figure describes the flowchart of the study.

\section{Percentage of surface hardness recovery (SHR)}

The final surface hardness (SHf) was measured at a $100 \mu \mathrm{m}$ distance from SHd, as described above. The mean of the three measurements was used to calculate the percentage of surface hardness recovery $(\% \mathrm{SHR}=[(\mathrm{SHf}-\mathrm{SHd}) / \mathrm{SHi}-\mathrm{SHd})] \times 100)$ for each block, then the mean \%SHR of 4 blocks for each volunteer $(n=10)$ was estimated.

\section{Statistical analysis}

Statistical analysis was performed with SigmaPlot version 12.3 (2011 Systat Software, Germany). The assumptions of normal distribution of errors were checked using Shapiro-Wilk test. Since assumptions were met, repeated measures ANOVA followed by Tukey's test were applied. The significance limit was set at $5 \%$.

\section{Results}

In the present study, all participants completed the in situ phase according to the protocol and no side effects were reported. All 40 blocks were submitted to surface hardness measure after each salivary exposure time.

Table shows the mean \%SHR for the different time periods. The null hypothesis was rejected, as longer periods promoted a progressive increases in SHR. Significant differences were found among 30 minutes, 1 - and 2 hours salivary exposure $(p<0.0001)$. There was no significant difference between 2 and 12 hours ( $p=0.641)$. 


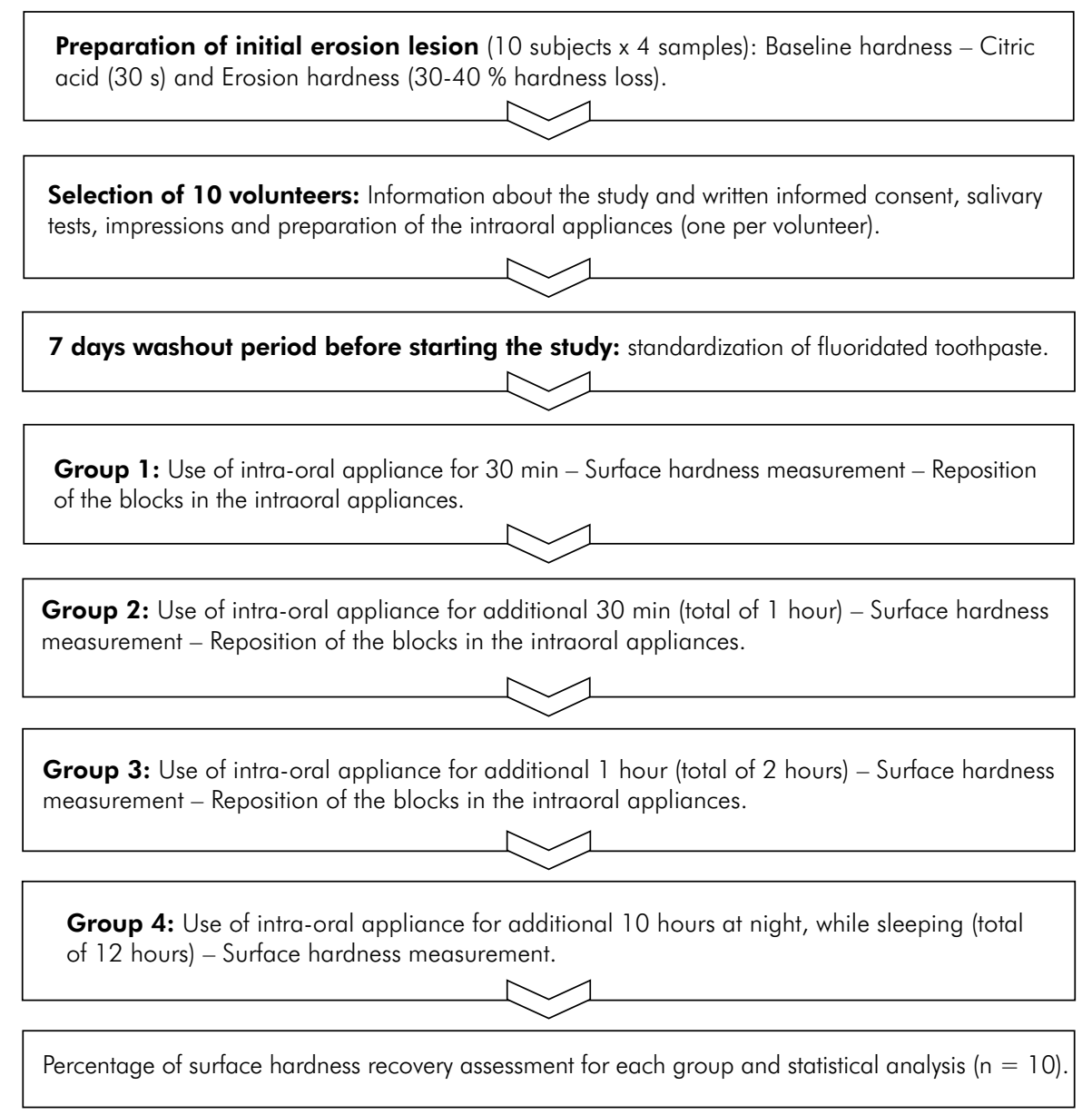

Figure. Flowchart of the study.

Table. Percentage of surface hardness recovery for the studied times of salivary exposure $(n=10)$.

\begin{tabular}{lcccc}
\hline Period of salivary exposure & 30 minutes & 1 hour & 2 hours & 12 hours \\
\hline Mean of \%SHR $( \pm$ SD) & $5.9( \pm 20.4)^{a}$ & $32.0( \pm 19.7)^{b}$ & $49.9( \pm 24.4)^{c}$ & $53.3( \pm 18)^{c}$ \\
\hline
\end{tabular}

Groups whose means are followed by distinct letters differ significantly. (ANOVA/Tukey's Test, p $<0.0001$ ).

\section{Discussion}

Saliva is the fluid that constantly flows through the oral cavity while awake. Thereby, saliva affects nearly all processes between enamel and the surrounding environment. ${ }^{12}$ In this study, no erosive cycling model was used, and only the salivary ability to reharden softened enamel after initial erosion was evaluated. The aim was to establish the most suitable period of time for intraoral device use to achieve maximum rehardening, before its submission to new erosive challenges on in situ cycling studies. The overnight effect of enamel rehardening by saliva during sleep, and therefore with reduced salivary flow, was also determined.

The erosive demineralization of enamel samples was performed in vitro by immersion of the samples in citric acid solution for 30 seconds, since the objective was to create an initial erosion lesion. The criterion used to ensure the softening but not loss of tissue was the visualization of baseline indentations after short-term acidic exposure. ${ }^{11}$ Surface hardness measurement has the advantages of directly measuring the physical stability of the rehardened enamel ${ }^{13}$ and 
of being a nondestructive method. ${ }^{14,15}$ This method has sufficient sensitivity to detect very early stages of enamel demineralization ${ }^{16}$, and is usually used for the evaluation of the rehardening potential of different substrates. ${ }^{17}$ In the present study the use of the same block to evaluate the rehardening effect of different salivary exposure times diminished the chance of bias from the enamel substrate variations. As observed in a pilot study, the variation in hardness among blocks during initial hardness selection was higher than the variations among study groups, hindering adequate conclusions. The present study design was possible because, for superficial hardness assessment, the same sample could be used for more than one measurement in a sequence. ${ }^{14}$

A possible limitation of this study is the period of time that the enamel blocks were outside the oral environment. Even though enamel blocks were kept hydrated with wet gauze, this might influence the acquired pellicle structure. However, since the main effect of the acquired pellicle is on erosion prevention, it probably had little impact in enamel remineralization. ${ }^{18}$ It must be emphasized that the time interval between the in situ salivary exposure periods necessary for measuring the surface hardness was standardized for all groups (3 hours). During these intervals, volunteers could eat, and therefore the subsequent tooth brushing was essential. Fluoridate toothpaste is widely recommended for patients to prevent erosion and dental caries. Hence, a fluoridate toothpaste was used to simulate clinical conditions as closely as possible. Despite the fact that fluoride might promote remineralization ${ }^{19}$, the residual fluoride from the fluoride-containing dentifrice did not protect enamel against erosion in an in situ protocol. ${ }^{20}$ In order to ensure no effect of fluoride in the remineralization process, a 1-hour time lapse was waited by volunteers between tooth brushing and reinsertion of the intraoral devices. This procedure was repeated before each study period.

Another possible limitation is the impact of tongue abrasion on eroded enamel. A previous study showed that the tongue exerts an abrasive effect on eroded enamel, partly removing the softened layer. ${ }^{21}$ In the present study, to minimize this effect, the volunteers were advised to avoid touching the blocks with the tongue. In addition, the time of oral appliance use was relatively short, diminishing the chance of unconscious tongue movements. Nevertheless, the superficial indentations $(25 \mathrm{~g} / 10 \mathrm{~s})$ made after the erosion procedure were evaluated after the in situ phase, and since they were still clearly observed, we assumed that the surface was very similar, showing minimal or none tongue abrasion.

The literature indicates that the remineralization and rehardening processes occur over a significantly long time scale ${ }^{22}$ and short remineralization times appear to provide only partial rehardening of softened enamel. ${ }^{13}$ In a previous study, after 24 hours of remineralization in artificial saliva, the stabilization of softened enamel surface was demonstrated by means of a normal level of resistance to ultrasonication. ${ }^{23}$ However, a 24-hour time period is of little clinical relevance since intraoral physical wear and periodic intake of acidic foods or drinks impose limits for remineralization. ${ }^{13}$ In the oral environment, remineralization periods range from $1-4 \mathrm{~h}$ at day time and from $6-8 \mathrm{~h}$ at night. ${ }^{24}$ However, during sleep, the salivary flow is reduced ${ }^{25}$ resulting in limited remineralization capacity.

The increasing time of salivary exposure promoted a progressive increase in the enamel surface hardness values. Periods of 30 minutes and 1 hour of salivary exposure in the oral cavity allowed a gain in surface hardness, suggesting that remineralization had occurred. Tooth remineralization has been described as a repair process by mineral deposition into the porous zone. ${ }^{26}$ After 2 hours of salivary action, surface hardness increased again, but it did not differ statistically from the 12-hour period, which was carried out overnight. These findings are of interest for in situ study protocols. Broadly, in situ models use two methodologies: removable appliances with intermittent or continuous intraoral use, or fixed appliances with continuous intraoral use. ${ }^{7}$ With intermittent use, the appliances are worn by subjects only under supervision and are removed from the mouth at nighttime. ${ }^{10}$ It is possible to assume that simplified in situ protocols and short-duration studies are most easily accepted by volunteers and therefore are likely conducted with higher fidelity to the established protocol. According to the presented results, considering only the remineralizing effect of saliva, the use of intraoral appliance during sleep 
is not justified. It is important to emphasize that even in studies with continuous use of the intraoral appliance, the last erosive challenge of the day would not be performed immediately before sleep. Thus in the present study, we tested the ability of saliva to improve, at night, rehardening that had occurred during the day; the rehardening ability during sleep was not evaluated.

Therefore, the minimum time interval to allow partial remineralization of acid-softened enamel between erosive challenges in in situ studies might be 2 hours. In addition, the standardization of 2-hour intervals and the removal of the intraoral appliance overnight might improve volunteers' collaboration, allowing more realistic and reliable results. However, more studies are needed to assess whether this degree of remineralization could protect the enamel from a subsequent acid attack.

\section{References}

1. Huysmans MC, Chew HP, Ellwood RP. Clinical studies of dental erosion and erosive wear. Caries Res. 2011;45(Suppl 1):60-8. doi:10.1159/000325947

2. Shellis RP, Addy M. The interactions between attrition, abrasion and erosion in tooth wear. Monogr Oral Sci. 2014;25:32-45. doi:10.1159/000359936

3. Rios D, Honório HM, Magalhães AC, Silva SM, Delbem AC, Machado MA et al. Scanning electron microscopic study of the in situ effect of salivary stimulation on erosion and abrasion in human and bovine enamel. Braz Oral Res. 2008;22(2):132-8. doi:10.1590/S1806-83242008000200007

4. Hara AT, Zero DT. The potential of saliva in protecting against dental erosion. Monogr Oral Sci. 2014;25:197-205. doi:10.1159/000360372

5. Zwier N, Huysmans MC, Jager DH, Ruben J, Bronkhorst EM, Truin GJ. Saliva parameters and erosive wear in adolescents. Caries Res. 2013;47(6):548-52. doi:10.1159/000350361

6. Jager DH, Vieira AM, Ligtenberg AJ, Bronkhorst E, Huysmans MC, Vissink A. Effect of salivary factors on the susceptibility of hydroxyapatite to early erosion. Caries Res. 2011;45(6):532-7. doi:10.1159/000331938

7. West NX, Davies M, Amaechi BT. In vitro and in situ erosion models for evaluating tooth substance loss. Caries Res. 2011;45(Suppl 1):43-52. doi:10.1159/000325945

\section{Conclusion}

In conclusion, considering the reparative effect of human saliva, the present study showed that 30 minutes, 1 and 2 hours of salivary exposure differed on the rehardening ability of acid-softened enamel. Two hours of salivary exposure seems to be appropriate for partial rehardening of the softened enamel surface between erosive challenges. The use of the intraoral appliance at night, including during sleep, did not improve enamel rehardening. This information might be used to guide future study designs on dental erosion.

\section{Acknowledgments}

The authors would like to gratefully acknowledge the volunteers who participated in this study and the Financial Research Support Foundation of the state of Sao Paulo (FAPESP) for financial support (Process $\left.n^{\mathrm{o}} 2012 / 20014-3\right)$.

8. Amaechi BT, Karthikeyan R, Mensinkai PK, Najibfard K, Mackey AC, Karlinsey RL. Remineralization of eroded enamel by a $\mathrm{NaF}$ rinse containing a novel calcium phosphate agent in an in situ model: a pilot study. Clin Cosmet Investig Dent. 2010;2:93-100. doi:10.2147/CCIDEN.S11844

9. Wiegand A, Attin T. Randomised in situ trial on the effect of milk and CPP-ACP on dental erosion. J Dent. 2014;42(9):1210-5. doi:10.1016/j.jdent.2014.07.009

10. West NX, Hughes JA, Parker D, Weaver LJ, Moohan M, De'Ath J et al. Modification of soft drinks with xanthan gum to minimise erosion: a study in situ. Br Dent J. 2004;196(8):478-81. doi:10.1038/sj.bdj.4811186

11. Young A, Tenuta LM. Initial erosion models. Caries Res. 2011;45(Suppl 1):33-42. doi:10.1159/000325943

12. Bardow A, Lykkeaa J, Qvist V, Ekstrand K, Twetman $\mathrm{S}$, Fiehn NE. Saliva composition in three selected groups with normal stimulated salivary flow rates, but yet major differences in caries experience and dental erosion. Acta Odontol Scand. 2014;72(6):466-73. doi:10.3109/00016357.2013.860621

13. Eisenburger M, Addy M, Hughes JA, Shellis RP. Effect of time on the remineralisation of enamel by synthetic saliva after citric acid erosion. Caries Res. 2001;35(3):211-5. doi:10.1159/000047458 
14. Wegehaupt FJ, Tauböck TT, Stillhard A, Schmidlin PR, Attin T. Influence of extra- and intra-oral application of CPP-ACP and fluoride on re-hardening of eroded enamel. Acta Odontol Scand. 2012;70(30):177-83. doi:10.3109/00016357.2011.600713

15. Attin T, Wegehaupt FJ. Methods for assessment of dental erosion. Monogr Oral Sci. 2014;25:123-42. doi:10.1159/000360355

16. Barbour ME, Rees JS. The laboratory assessment of enamel erosion: a review. J Dent. 2004;32(8):591-602. doi:10.1016/j.jdent.2004.05.001

17. Ionta FQ, Mendonça FL, Oliveira GC, Alencar CR, Honório HM, Magalhães AC et al. In vitro assessment of artificial saliva formulations on initial enamel erosion remineralization. J Dent. 2014;42(2):175-9. doi:10.1016/j.jdent.2013.11.009

18. Hara AT, Ando M, González-Cabezas C, Cury JA, Serra $\mathrm{MC}$, Zero DT. Protective effect of the dental pellicle against erosive challenges in situ. J Dent Res. 2006;85(7):612-6. doi:10.1177/154405910608500706

19. Huysmans MC, Young A, Ganss C. The role of fluoride in erosion therapy. Monogr Oral Sci. 2014;25:230-43. doi:10.1159/000360555

20. Magalhães AC, Rios D, Martinhon CC, Delbem AC, Buzalaf MA, Machado MA. The influence of residual salivary fluoride from dentifrice on enamel erosion: an in situ study. Braz Oral Res. 2008;22(1):67-71. doi:10.1590/S1806-83242008000100012

21. Gregg T, Mace S, West NX, Addy M. A study in vitro of the abrasive effect of the tongue on enamel and dentine softened by acid erosion. Caries Res. 2004;38(6):557-60. doi:10.1159/000080586

22. Fowler C, Willson R, Rees GD. In vitro microhardness studies on a new anti-erosion desensitizing toothpaste. J Clin Dent. 2006;17(4):100-5.

23. Eisenburger M, Hughes J, West NX, Shellis RP, Addy M. The use of ultrasonication to study remineralisation of eroded enamel. Caries Res. 2001;35(1):61-6. doi:10.1159/000047433

24. Lippert F, Parker DM, Jandt KD. In vitro demineralization/remineralization cycles at human tooth enamel surfaces investigated by AFM and nanoindentation. J Colloid Interface Sci. 2004;280(2):442-8. doi:10.1016/j.jcis.2004.08.016

25. Schneyer LH, Pigman W, Hanahan L, Gilmore RW. Rate of flow of human parotid, sublingual and submaxillary secretions during sleep. J Dent Res. 1956;35(1):109-14. doi:10.1177/00220345560350010301

26. Imfeld T. Prevention of progression of dental erosion by professional and individual prophylactic measures. Eur J Oral Sci. 1996;104(2):215-20. doi:10.1111/j.1600-0722.1996.tb00070.x 